\title{
ATIVIDADE DO EXTRATO, FRAÇÕES E SUBSTÂNCIAS ISOLADAS DAS CAS- CAS DE Geissospermum vellosii ALLEMÃO (APOCYNACEAE) SOBRE Artemia salina LEACH
}

\section{ACTIVITY OF EXTRACT, FRACTIONS AND ISOLATED SUBSTANCES OF THE BARKS FROM Geissospermum vellosii ALLEMÃO (APOCYNACEAE) ON Arte- mia salina LEACH}

DIAS, Josiane de F.G. ${ }^{1}$; KALEGARI, Milena1; MIGUEL, Marilis D. ${ }^{1}$; ZANIN, Sandra M.W. ${ }^{\text {; }}$ LORDELLO, Ana Luisa L. ${ }^{2}$; SANTOS, Luciana M. Dos ${ }^{3}$, SENS, Rafaele C. V. ${ }^{3}$,WUNDER, Paulo R. ${ }^{1}$; MIGUEL, Obdúlio G. ${ }^{1}$

1UFPR, Programa de Pós Graduação em Ciências Farmacêuticas, Campus Jardim Botânico, 80210170, Curitiba, Brasil. email: jodias @ ufpr.br

2UFPR, Departamento de Química, Centro Politécnico, 81531-990, Curitiba, Brasil

${ }^{3}$ UFPR, Departamento de Farmácia, Campus Jardim Botânico, 80210-170, Curitiba, Brasil

\section{RESUMO:}

O extrato, frações e substâncias isoladas das cascas de G. vellosii foram submetidos ao teste de bioatividade sobre $A$. salina. Os dados foram analisados pelo método Probitos e determinados os valores de $\mathrm{DL}_{50}$ e $95 \%$ de intervalos de confiança. As amostras foram consideradas ativas quando $\mathrm{DL}_{50}<1000 \mu \mathrm{g} \cdot \mathrm{mL}^{-1}$. Todas as amostras testadas demonstraram bioatividade contra $A$. salina, destacando-se $\mathrm{F} 4$ e $\mathrm{F} 9$, as quais apresentaram valores de $\mathrm{DL}_{50}$ de 7,60 e $29,48 \mu \mathrm{g} \cdot \mathrm{mL}^{-1}$, respectivamente. Os resultados demonstram o potencial biotecnológico da espécie, pois segundo literatura este bioensaio pode ser utilizado como avaliação prévia de possível atividade antitumoral.

Palavras-chave: Geissospermum, Artemia, bioatividade.

\section{ABSTRACT:}

The extract, fractions and isolated substances of the barks from $G$. vellosii were submitted to the bioactivity test on $A$. salina. The data were analyzed by the Probitos' method and certain the values of $\mathrm{DL}_{50}$ and $95 \%$ of trust intervals. The samples were considered active when $\mathrm{DL}_{50}<1000 \mu \mathrm{g} \cdot \mathrm{mL}^{-1}$. All of the tested samples demonstrated bioactivity against $A$. salina, standing out $\mathrm{F} 4$ and $\mathrm{F} 9$, which presented values of $\mathrm{DL}_{50} 7.60$ and $29.48 \mu \mathrm{g} \cdot \mathrm{mL}^{-1}$, respectively. The results demonstrate the biotechnological potential of the species, because according to literature this bioessay can be used as previous evaluation of possible antitumoral activity.

Key words: Geissospermum, Artemia, bioactivity.

\section{INTRODUÇÃO}

A espécie Geissospermum vellosii é conhecida popularmente como Pau Pereira (MANSKE \& HOLMES, 1952), Câmara-de-Bilro, Ubá-Açu, Pau-Forquilha, Canudo-Amargoso, Tringuaaba, Pau-Pente, Câmara-do-Mato, Pinguaciba, Pereiro (CORRÊA, 1974) e Maria Congo (BERTANI et al., 2005). Apresenta os sinônimos Geissospermum leave, Miers, Geissospermum vellozii, Fr. Allem (INDEX KEWENSIS, 1895), Tabernaemontana laevis (CORRÊA, 1974; (INDEX KEWENSIS, 1913) e Geissospermum laevis, Miers (CORRÊA, 1974). 
As substâncias geissospermina, pereirina, velosina (MANSKE \& HOLMES, 1952), flavopereirina (HUGHES \& RAPOPORT, 1958), velosimina, velosiminol, geissolosimina (RAPOPORT \& MOORE, 1962) e geissovelina (MOORE e RAPOPORT, 1973) foram isoladas da referida espécie. O extrato etanólico de $G$. vellosii apresenta atividade contra Plasmodium vinckei (BERTANI et al., 2005; MUÑOZ et al., 2000) e atividade antinociceptiva (WERNER et al., 2009). A flavopereirina apresenta atividade contra Plasmodium falciparum (SOMEL \& YAMADA, 2004) e atividade antitumoral (BELJANSKI \& BELJANSKI, 1982), assim como seu derivado denominado PB-100 (BELJANSKI, 2000). A geissospermina apresenta ação hipotensora e ação depressora do Sistema Nervoso Central (AUROUSSEAU, 1960; AUROSSEAU, 1961), foi classificada como veneno paralisante vascular (CORRÊA, 1974) e apresenta ação relaxante (TANAE et al., 1999; TANAE et al., 2004).

O ensaio de bioatividade com Artemia salina é utilizado por ser rápido, de baixo custo (MEYER et al., 1982), não requerer ambiente asséptico, utilizar grande número de organismos para validação estatística, não requerer equipamentos especiais e utilizar quantidade de amostra relativamente pequena (2-20 mg) (MCLAUGHLIN et al., 991). Alguns trabalhos tentam correlacionar a toxicidade sobre $A$. salina com atividades como antifúngica, viruscida, antimicrobiana, tripanossomicida (MACRAE et al., 1988), parasiticida (CHAN-BACAB et al., 2003) e antitumoral (RUPPRECHT et al., 1990; SIQUEIRA et al., 2001).

Nesta perspectiva, este trabalho teve como objetivo testar extrato, frações e substâncias isoladas de G. vellosii sobre A. salina.

\section{MATERIAL E MÉTODOS}

\subsection{Obtenção do material vegetal}

Cascas de G. vellosii foram colhidas em novembro de 2002. O material vegetal foi identificado por comparação com exsicata registrada no Museu Botânico de Curitiba sob número 36060 como Geissospermum vellosii. Cascas moídas de G. vellosii foram submetidas à extração em Soxhlet em etanol absoluto até esgotamento total obtendo-se o extrato bruto etanólico, o qual foi fracionado em extrator de Soxhlet modificado (CARVALHO et al, 2009) com solventes de polaridade crescente, obtendo-se as frações diclorometano, acetato de etila, butanol e hidroalcóolica. A fração diclorometano foi acrescida de duas partes de sílica gel em relação ao seu peso e foi cromatografada por meio de coluna com sílica gel utilizando fases móveis apropriadas com uma vazão de $1 \mathrm{~mL}$ por minuto. Foram coletados frascos de $20 \mathrm{~mL}$ que foram reunidos segundo monitoramento por cromatografia em camada delgada. Após reunião dos frascos, estes foram levados à secura, lavados com benzina e filtrados em funil de vidro sinterizado, obtendo-se substâncias denominadas preliminarmente de F4, F9 e F17. 


\subsection{Ensaio de bioatividade com Artemia salina}

O ensaio foi realizado segundo Meyer et al. (1982) com amostras do extrato etanólico, frações diclorometano, acetato de etila, butanol, hidroalcóolica e substâncias F4, F9 e F17. Foram pesados 20 mg de cada amostra e adicionado clorofórmio em quantidade suficiente para obter a concentração inicial de $10 \mathrm{mg} \cdot \mathrm{mL}^{-1}$. Desta solução foram transferidos 5,50 e $500 \mathrm{~mL}$ para tubos de ensaio correspondendo respectivamente a 10, 100 e $1000 \mathrm{mg} \cdot \mathrm{mL}^{-1}$, em triplicata. O solvente foi evaporado por 12 horas (MCLAUGHLIN et al., 1991). Após a eclosão dos ovos, foram transferidas 10 larvas de Artemia salina para cada tubo de ensaio contendo as amostras e para os tubos controles. Os controles negativos consistiram de tubos com clorofórmio, o qual foi evaporado juntamente com as amostras, e de tubos vazios. O controle positivo foi sulfato de quinidina. $O$ volume de todos os tubos foi ajustado com água do mar artificial para $5 \mathrm{~mL}$. Após 24 horas foi realizada a contagem das larvas mortas e vivas, com auxílio de lupa e iluminação incandescente. Os dados foram analizados com o método estatístico Probitos (FINNEY, 1971) e determinados os valores de DL e 95\% de intervalos de confiança. As frações foram consideradas ativas quando $\mathrm{DL}_{50}^{50}$ foi menor que $1000 \mathrm{mg} \cdot \mathrm{mL}^{-1}$ (MEYER et al., 1982).

\section{RESULTADOS E DISCUSSÃO}

Ao analisar os resultados obtidos (Tabela 1), verifica-se que todas as amostras apresentaram DL menor que $1000 \mathrm{mg} \cdot \mathrm{mL}^{-1}$. Os menores $\mathrm{DL}$ foram verificados pelas substâncias isolađ̆as F4, F9 e F17, as quais preliminarmente foram identificadas como alcalóides.

TABELA 1 -AVALIAÇÃO DAATIVIDADE DE AMOSTRAS DE G. vellosii SOBRE Artemia salina

\begin{tabular}{c|c|c}
\hline Amostra & $\mathbf{D L}_{\mathbf{5 0}}\left(\boldsymbol{\mu} \mathbf{g} \cdot \mathbf{m L}^{-1}\right)$ & Intervalo de confiança de $\mathbf{9 5} \mathbf{\%}\left(\boldsymbol{\mu g} \cdot \mathbf{m L}^{-1}\right)$ \\
\hline Extrato Bruto Etanólico & 398,11 & $66,05-2399,68$ \\
\hline Fração Diclorometano & 251,19 & $195,18-323,25$ \\
\hline F4 & 7,60 & $4,67-12,34$ \\
\hline F9 & 29,48 & $21,51-37,71$ \\
\hline F17 & 232,63 & $174,80-309,59$ \\
\hline Fração Acetato de Etila & 173,65 & $93,37-322,94$ \\
\hline Fração Butanol & 146,70 & $28,13-765,90$ \\
\hline Fração Hidroalcoolica & 439,40 & $240,28-803,53$ \\
\hline Sulfato de Quinidina & 50,12 & $35,80-70,16$
\end{tabular}

Ao comparar os resultados obtidos com o resultado de outros autores, verifica-se que os alcalóides possuem toxicidade sobre Artemia salina (MARTínEZ et al, 1997; SIQUEIRA et al., 1998; SIQUEIRA et al., 2001) e estes resultados podem correlacionar a espécie com outras atividades biológicas (MACRAE et al., 1988; RUPPRECHT et al., 1990; SIQUEIRA et al., 2001; CHAN-BACAB et al., 2003).

Mais ensaios serão conduzidos com a referida espécie para verificação de out- 
ras atividades biológicas.

\section{REFERÊNCIAS}

AUROUSSEAU, M.M. Etude pharmacodynamique de la geissospermine alcaloide principal du Geissospermum laeve (Vellozo) Baillon (Apocynacées). Annales Pharmaceutiques Francaises, v.19, n.2, p.104-116, 1960.

AUROUSSEAU, M.M. Etude pharmacodynamique de la geissospermine alcaloide principal du Geissospermum laeve (Vellozo) Baillon (Apocynacées). Annales Pharmaceutiques Francaises, v.19, n.3, p.175-189, 1961.

BELJANSKI, M.; BELJANSKI, M.S. Selective inhibition of in vitro synthesis of cancer DNA by alkaloids of $\beta$-carboline class. Pathobiology, v.50, n.2, p. 79-87, 1982.

BELJANSKI, M. The anticancer agent PB-100, selectively active on malignant cells, inhibits multiplication of sixteen malignat cells lines, even multidrug resistant. Genetics and Molecular Biology, v.23, n. 1, p. 29-33, 2000.

BERTANI, S.; BOURDY, G.; LANDAU, I.; ROBINSON, J.C.; ESTERRE Ph.; DEHARO, E. Evaluation of French Guiana traditional antimalarial remedies. Journal of Ethnopharmacology, v.98, p. 45-54, 2005.

CARVALHO, J.L.S.; CUNICO, M.M.; DIAS, J.F.G.; MIGUEL, M.D.; MIGUEL, O.G.

Termoestabilidade de processos extrativos de Nasturtium officinale R. Br., Brassicaceae por sistema Soxhlet modificado. Quimica. Nova, v. 32, n. 4, p.1031-1035, 2009.

CHAN-BACAB, M.J. et al. Variation of leishmanicidal activity in four populations of Urechites andrieuxii. Journal of Ethnopharmacology, v. 86, p. 243-247, 2003.

CORRÊA, M.P. Dicionário das plantas úteis do Brasil e das exóticas cultivadas. Rio de Janeiro: Ministério da Agricultura, 1974.

FINNEY, D.J. Probit Analysis. Cambridge University Press: Cambridge, 1971.

HUGHES, N.A.; RAPOPORT, H. Flavopereirina, an alkaloid from Geissospermum vellosii. Journal American of Chemical Society, n. 80, v.7, p. 1604-1609, 1958.

INDEX KEWENSIS. Oxford University Press, 1895, p.179.

INDEX KEWENSIS. Oxford University Press, 1913, p.95.

MANSKE, R.H.F.; HOLMES, H.L. The alkaloids chemistry and phisiology. New York: Academic Press Inc, 1952. p. 369-481.

MARTÍNEZ; J.L.; TORRES, R.; MORALES, M.A. Hypotensive effect of O-methyli- 
sothalicberine, a bisbenzylisoquinoline alkaloid isolated from Berberis chilensis on normotensive rats. Phytotherapy Research, v.11, n.3, p.246-248, 1997.

MCLAUGHLIN, J.L.; CHANG, C.J.; SMITH, D.L. "Bendh-Top" bioassays for the discovery of bioactive natural products: an update. In: RAHMAN, A.R. (Ed.) Studies in Natural Products Chemistry. Amsterdam: Elsevier Science Publishers B.V., p. 383409, 1991.

MACRAE, W.D.; HUDSON, J.B.; TOWERS, G.H. Studies on the pharmacological activity of Amazonian Euphorbiaceae. Journal of Ethnopharmacology, v. 22, p. 14372, 1988.

MEYER, B.N. et al. Brine Shrimp: a convenient general bioassay for active plant constituents. Planta medica, v. 45, p. 31, 1982.

MOORE, R.E.; RAPOPORT, H. Geissovelline, a new alkaloid from Geissospermum vellosii. Journal of Organic Chemistry, v.38, n.2, 215-230, 1973.

MUÑOZ,V.; SAUVAIN, M.; BOURDY, G.; CALLAPA, J.; BERGERON, S.; ROJAS, I.; BRAVO, J.A.; BALDERRAMA, L.; ORTIZ, B.; GIMENEZ, A.; DEHARO,E. A search for natural bioactive compounds in Bolivia through a multidisciplinary approach Part I Evaluation of the antimalarial activity of plants used by the Chacobo Indians. Journal of Ethnopharmacology, v. 69, p.127-137, 2000.

RAPOPORT, H.; MOORE, R.E. Alkaloids of Geissospermum vellosii. Isolation and structures determinations of vellosimine, vellosiminol and geissolosimine. The Journal of Organic Chemistry, v.27, n.9, p. 2981-2985, 1962.

RUPPRECHT, J.K.; HUI, Y.H.; MCLAUGHLIN, J.L. Annonaceous acetogenins: a review. Journal of Natural Products,v. 53, p. 237-278, 1990.

SIQUEIRA, J.M.; BOMM, M.D.; PEREIRA, N.F.G.; GARCEZ, W.S.G.; BOAVENTURA, M.A.D. Estudo fitoquímico de Unonopsis lindmanii - ANNONACEAE, biomonitorado pelo ensaio de toxicidade sobre a Artemia salina LEACH. Química Nova, v.21, n.5, p. 557-559, 1998.

SIQUEIRA, J.M.; ZIMINIANI, M.G.; RESENDE, U.M.; BOAVENTURA, M.A.D. Estudo fitoquímico das cascas do caule de Duguetia glabriuscula - Annonaceae, biomonitorado pelo ensaio de toxicidade frente a Artemia salina LEACH. Química Nova, v. 24, n. 2, p.185-187, 2001.

SOMEL, M.; YAMADA, F. Simple indole alkaloids and those with a nonrearranged monoterpenic unit. Natural Product Reports, v.21, p.278-311, 2004.

TANAE, M.M.; MOTIDOME, M.; VALLIN, A.A.C..; CARDOSO E.M.; SOUCCAR, C.; LIMA-LANDMAN, M.T.R. Liberação da geissospermina, alcalóide isolado do pau-pereira (Geissospermum vellosii) no músculo esquelético. In: Reunião anual da FESBE, 14, 1999, Caxambú. Anais da XIV Reunião anual da FESBE. Caxambú, 1999, p. 387. 
TANAE, M.M.; GHEDINI, P.C.; SOUCCAR, C.; MOTIDOME, M.; LAPA, A.J.; LIMA-LANDMAN, M.T.R. Interação de Geissospermina, alcalóide isolado do pau pereira (Geissospermum laeve), com receptores nicotínicos musculares. In: Simpósio de Plantas Medicinais do Brasil, 18, 2004, Manaus. Livro de Resumos. Manaus, 2004, p.431.

WERNER, J.A.T., OLIVEIRA, S.M.; MARTINS, L.M.; DIAS, J.F.G.; LORDELLO, A.L.L.; MIGUEL, O.G.; ROYES, L.F.; FERREIRA, J.; SANTOS, A.R.S. Evidence for a role of 5-HT1A receptor on antinociceptive action from Geissospermum vellosii. Journal of Ethnopharmacology , v. 125, p. 163-169, 2009. 